\title{
Adrenal, thyroid and gonadal axes are affected at high altitude
}

\author{
M von Wolff' ${ }^{1, *}$, C T Nakas ${ }^{2,3, *}$, M Tobler ${ }^{1,4}$, T M Merz ${ }^{5}$, M P Hilty ${ }^{6}$, J D Veldhuis ${ }^{7}$, A R Huber ${ }^{8}$ and J Pichler Hefti ${ }^{4, *}$ \\ ${ }^{1}$ Division of Gynaecological Endocrinology and Reproductive Medicine, University Women's Hospital, Bern University Hospital, University of Bern, \\ Bern, Switzerland \\ ${ }^{2}$ University Institute of Clinical Chemistry, Inselspital, Bern University Hospital, University of Bern, Bern, Switzerland \\ ${ }^{3}$ Laboratory of Biometry, University of Thessaly, Volos, Greece \\ ${ }^{4}$ Division of Pneumology, Inselspital, Bern University Hospital, University of Bern, Bern, Switzerland \\ ${ }^{5}$ Division of Intensive Care Medicine, Inselspital, Bern University Hospital, University of Bern, Bern, Switzerland \\ ${ }^{6}$ Intensive Care Unit, University Hospital, Zurich, Switzerland \\ ${ }^{7}$ Endocrine Research Unit, Department of Internal Medicine, Mayo School of Graduate Medical Education, Centre for Translational Science Activities, \\ Mayo Clinic, Rochester, New York, USA \\ ${ }^{8}$ Centre for Laboratory Medicine, Cantonal Hospital, Aarau, Switzerland
}

Correspondence should be addressed to M von Wolff: Michael.vonWolff@insel.ch

*(M von Wolff, C T Nakas and J Pichler Hefti contributed equally to this work)

\begin{abstract}
Humans cannot live at very high altitude for reasons, which are not completely understood. Since these reasons are not restricted to cardiorespiratory changes alone, changes in the endocrine system might also be involved. Therefore, hormonal changes during prolonged hypobaric hypoxia were comprehensively assessed to determine effects of altitude and hypoxia on stress, thyroid and gonadal hypothalamus-pituitary hormone axes. Twenty-one male and 19 female participants were examined repetitively during a high-altitude expedition. Cortisol, prolactin, thyroid-stimulating hormone (TSH), fT4 and fT3 and in males follicle-stimulating hormone (FSH), luteinizing hormone (LH) and total testosterone were analysed as well as parameters of hypoxemia, such as $\mathrm{SaO}_{2}$ and $\mathrm{paO}_{2}$ at $550 \mathrm{~m}$ (baseline) $(n=40)$, during ascent at $4844 \mathrm{~m}(n=38)$, $6022 \mathrm{~m}(n=31)$ and $7050 \mathrm{~m}(n=13)$, at $4844 \mathrm{~m}(n=29)$ after acclimatization and after the expedition $(n=38)$. Correlation analysis of hormone concentrations with oxygen parameters and with altitude revealed statistical association in most cases only with altitude. Adrenal, thyroid and gonadal axes were affected by increasing altitude. Adrenal axis and prolactin were first supressed at $4844 \mathrm{~m}$ and then activated with increasing altitude; thyroid and gonadal axes were directly activated or suppressed respectively with increasing altitude. Acclimatisation at $4844 \mathrm{~m}$ led to normalization of adrenal and gonadal but not of thyroid axes. In conclusion, acclimatization partly leads to a normalization of the adrenal, thyroid and gonadal axes at around $5000 \mathrm{~m}$. However, at higher altitude, endocrine dysregulation is pronounced and might contribute to the physical degradation found at high altitude.
\end{abstract}

Key Words
- cortisol
- prolactin
- TSH
- $\mathrm{fT} 3$
- fT4
- follicle-stimulation
hormone
- luteinizing hormone
- testosterone
- altitude
- hypobaric hypoxia

Endocrine Connections (2018) 7, 1081-1089

\section{Introduction}

Humans have populated a wide variety of climatic and geographical regions of the world. However, altitudes of around $5000 \mathrm{~m}$ are only permanently colonized in rare cases, for example mining communities in the Andes. This fact can be explained not only by the hostile climate, but also by the inadequate physiological capacity of the
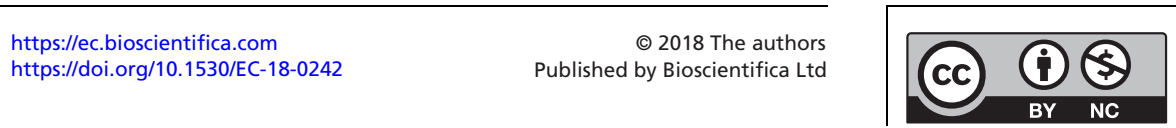
human organism to adapt to this altitude in the long term or to reproduce in this environment.

A longer-term existence at high altitude requires a variety of cardiorespiratory and metabolic changes (Luks, 1985), a compensated metabolism and, in the case of permanent settlement at higher altitudes, adequate fertility.

This raises the question of whether the endocrine system, which plays a central role in the regulation of almost all bodily functions, becomes significantly dysregulated above a certain altitude.

Several studies have already investigated various hormone concentrations at high altitude $(1,2,3,4,5,6$, $7,8,9,10)$.

However, the study groups were mostly small, limited to men, were only carried out at altitudes of up to $5000 \mathrm{~m}$ above sea level or the study design was not ideal. This might be the reason for the often heterogeneous results of these studies, which have not allowed a conclusive statement about the effect of altitude on the endocrine system to be made up to now.

The aim was therefore to perform an observational study in a large collective, with both male and female subjects at various altitudes up to $7000 \mathrm{~m}$ under standardized conditions and to investigate the changes in the adrenal, thyroid and gonadal hypothalamus-pituitary end-organ axes.

\section{Materials and methods}

\section{Setting}

This was a prospective observational cohort study conducted during the Swiss High Altitude Medical Research Expedition Himlung Himal 2013 (11). The aim of this project was to investigate the cerebral, cardiovascular, pulmonary and humoral adaptation to prolonged hypobaric hypoxia during an ascent to Mount Himlung Himal (7126m) in Nepal.

Baseline and post expedition testing was performed in two groups in Berne, Switzerland $(550 \mathrm{~m}) 8$ and 9 weeks before the start of the expedition ( $\mathrm{PR}=$ Pre expedition). For logistical reasons, the journey as well as the expedition testing were planned in two groups with identical ascent profiles. Comprehensive medical assessments were performed in Base Camp (BC1) at $4844 \mathrm{~m}$ on day 7, in Camp 2 (C2) $(6022 \mathrm{~m})$ on day 13 and in Camp 3 (C3) $(7050 \mathrm{~m})$ on day 23/24 respectively. An additional measurement was carried out at Base Camp on day
19/20 (BC2) to study the effect of acclimatization. A final analysis was performed 4-5 weeks after the expedition at $550 \mathrm{~m}$ (PO=Post-expedition).

The number of subjects included in this study was restricted due to logistical and safety concerns. Forty healthy subjects (21 male, 19 female), with basic mountaineering experience and aged between 18 and 70 years, were included in the trial. Exclusion criteria consisted of the presence of any neurologic, cardiac or respiratory disease, diabetes mellitus type I or II or the need for any regular medication, particularly thyroid hormones, corticosteroids and other medication, which could possibly affect hormone analysis. In addition, subjects were excluded if a known susceptibility for high altitude sickness was present. Susceptibility was defined as a history of severe acute mountain sickness, high altitude cerebral oedema (HACE) or high altitude pulmonary oedema (HAPE) after a rapid ascent ( $<3$ nights) at altitudes below $3500 \mathrm{~m}$.

The study was approved by the Cantonal Ethics Committee (KEK 226/12) and has been registered on clinicaltrials.gov (NCT01953198). Consent has been obtained from each patient after full explanation of the purpose and nature of all procedures used.

\section{Measurements}

Arterial blood gas sampling and oxygen transport parameters: Arterial blood was aspirated using a blood gas sampler (safe PICO Aspirator, Radiometer Medical, Denmark) and immediate analysis was done with a pocket analyser (Epoc, Alere, Wadenswil, Switzerland) to assess arterial oxygen saturation $\left(\mathrm{SaO}_{2}\right)$, partial pressure for $\mathrm{O}_{2}$ $\left(\mathrm{paO}_{2}\right)$ and haemoglobin $(\mathrm{Hb})$.

Blood sampling: At each study site, fasting blood samples were taken between 07.15 and $09.00 \mathrm{~h}$. The samples were collected in sterile serum tubes (Sarstedt, Sevelen, Switzerland). All samples were immediately centrifuged for $10 \mathrm{~min}$ at $2000 \boldsymbol{g}$ (EBA 20, Hettich AG, Bäch, Switzerland). Aliquots were frozen to $-40^{\circ}$ to $-60^{\circ} \mathrm{C}$ on-site and stored at $-80^{\circ} \mathrm{C}$ thereafter.

Cortisol, prolactin, thyroid-stimulating hormone (TSH), free tetraiodothyronine (fT4), free triiodothyronine (fT3), follicle-stimulating hormone (FSH), luteinizing hormone (LH) and total testosterone (testosterone) were analysed. FSH, LH and testosterone were only evaluated in males due to the menstrual-dependent fluctuations in females.

Hormone analysis: Cortisol levels were determined using a competitive immunoassay based on a 
chemiluminescence technology (Advia Centaur Cortisol Assay, Siemens) on an Advia Centaur System (Siemens). Reference values were 140-700 nmol/L. Quantitative assessment of prolactin (PRL) and LH levels was performed on Dimension Vista R System (Siemens) using a sandwich chemiluminescent immunoassay (Flex Reagent Cartridge, Siemens). The respective reference intervals for PRL were in adult females $2.2-28 \mu \mathrm{g} / \mathrm{L}$ and in adult males $2.7-17 \mu \mathrm{g} / \mathrm{L}$. The same method was used for the quantitative determination of TSH, fT3 and fT4. Reference values for fT3 were 3.2-6.2 pmol/L, for fT4 9.9-19.3 pmol/L and for TSH 0.4-4.0 mIU/L. Testosterone was assessed with a solidphase chemiluminescent immune assay (IMMULITE 2000 System, Siemens). Respective reference ranges in men aged $\leq 60$ years were $7-32 \mathrm{nmol} / \mathrm{L}$ and in men $>60$ years $5-32 \mathrm{nmol} / \mathrm{L}$.

FSH was measured using a homogeneous sandwich chemiluminescent immunoassay (FSH Flex Reagent Cartridge, Siemens) on a Dimension Vista System. Reference values in adult males are $0.7-11 \mathrm{IU} / \mathrm{L}$.

\section{Statistics}

Clinical data are presented as mean and standard deviation (s.D.). Standard errors (s.e.) are also presented when CIs are given.

To assess the effects of altitude and hypoxia on a selected group of hormones, generalized estimating equation modelling was employed in order to efficiently accommodate the longitudinal measurements during the expedition. Sidak adjustment was used for multiple pairwise (post hoc) comparisons. $P$ values less than 0.05 were considered statistically significant. Adjustment for sex, $\mathrm{SaO}_{2}$ or $\mathrm{CaO}_{2}$ was performed where needed as assessed by initial univariate analyses. Data analysis was performed using STATA 13.1, (STATA Corp.).

\section{Results}

Of the 40 subjects, one met an exclusion criterion during the baseline testing and was therefore excluded from the following expedition. Number of subjects was lower at high altitudes but the average age of the analysed subjects was not different. $\mathrm{SaO}_{2}, \mathrm{paO}_{2}$ and $\mathrm{Hb}$ varied significantly at different altitudes (Table 1 ).

Hormone concentrations were assessed for the effect of hypoxia parameters and altitude. In most cases, only altitude resulted in a significant effect (Tables 2, 3, 4, 5, $6,7,8,9$ and 10). Prolactin concentrations were also sex dependent $(P=0.015)$. fT3 $(P=0.024)$ and testosterone $(P=0.028)$ were $\mathrm{SaO}_{2}$ dependent and TSH $(P=0.011)$ was $\mathrm{pO}_{2}$ dependent. Accordingly, in the modelling approach, we adjusted for these parameters while assessing the effect of altitude. Average hormone concentrations stayed within the normal ranges at all altitudes; however, in individual cases concentrations reached pathological concentrations as shown in Figs 1 and 2 for cortisol and fT4.

Hormone axes were affected by altitude as follows:

\section{Adrenal axis and prolactin}

Cortisol concentrations revealed a U-shaped pattern with increasing altitude (Fig. 1 and Table 2). Concentrations first decreased significantly from $474 \pm 163 \mathrm{nmol} / \mathrm{L}$ before the expedition (baseline) to $369 \pm 106$ at $4844 \mathrm{~m}$ and increased 1.7 -fold thereafter to reach $643 \pm 91 \mathrm{nmol} / \mathrm{L}$ at $7050 \mathrm{~m}$ altitude. Acclimatization at $4844 \mathrm{~m}$ led to normalization of cortisol concentration compared to baseline. These changes were neither sex nor $\mathrm{SaO}_{2}$ or $\mathrm{paO}_{2}$ dependent.

Changes in prolactin (Table 3 ) concentrations were less pronounced. Prolactin also dropped significantly at $4844 \mathrm{~m}$ and increased at $6022 \mathrm{~m}$, but the increase was not significant. However, prolactin also normalized at $4844 \mathrm{~m}$ after acclimatization as prolactin concentration

Table 1 Basic characteristics of subjects.

\begin{tabular}{l}
\hline Subjects total, $n$ \\
Females, $n$ \\
Males, $n$ \\
Age total (mean \pm s.D.) \\
Age females (mean \pm S.D.) \\
Age males (mean \pm S.D.) \\
$\mathrm{SaO}_{2}$ (mean \pm S.D.) \\
$\mathrm{paO}_{2}$ (mean \pm S.D.) \\
$\mathrm{Hb}$ (mean \pm S.D.)
\end{tabular}

\begin{tabular}{c}
\multicolumn{1}{c}{$\mathbf{P R}$} \\
\hline 40 \\
19 \\
21 \\
$45.56 \pm 12.06$ \\
$40.94 \pm 10.96$ \\
$49.52 \pm 11.78$ \\
$97.57 \pm 0.79$ \\
$95.35 \pm 7.87$ \\
$14.80 \pm 0.93$ \\
\hline
\end{tabular}

\begin{tabular}{c}
\hline \multicolumn{1}{c}{ BC1 } \\
\hline 38 \\
17 \\
21 \\
$46.08 \pm 11.78$ \\
$41.82 \pm 10.62$ \\
$49.52 \pm 11.78$ \\
$83.78 \pm 4.59$ \\
$44.18 \pm 4.52$ \\
$15.35 \pm 0.90$
\end{tabular}

\begin{tabular}{c}
\hline \multicolumn{1}{c}{$\mathbf{2}$} \\
\hline 31 \\
14 \\
17 \\
$46.39 \pm 12.36$ \\
$42.00 \pm 11.25$ \\
$50.00 \pm 12.37$ \\
$72.71 \pm 8.83$ \\
$34.34 \pm 4.99$ \\
$16.03 \pm 1.10$
\end{tabular}

\begin{tabular}{c}
\hline BC2 \\
\hline 29 \\
13 \\
16 \\
$47.18 \pm 11.90$ \\
$43.75 \pm 10.23$ \\
$49.75 \pm 12.71$ \\
$88.59 \pm 2.34$ \\
$50.98 \pm 3.57$ \\
$17.03 \pm 0.89$ \\
\hline
\end{tabular}

\begin{tabular}{c}
\hline $\mathbf{C 3}$ \\
\hline 13 \\
5 \\
8 \\
$43.85 \pm 13.74$ \\
$37.20 \pm 13.66$ \\
$48.00 \pm 12.87$ \\
$68.8 \pm 9.65$ \\
$30.38 \pm 4.41$ \\
$17.5 \pm 1.00$ \\
\hline
\end{tabular}

\begin{tabular}{c} 
PO \\
\hline 38 \\
17 \\
21 \\
$45.37 \pm 12.16$ \\
$40.24 \pm 10.86$ \\
$49.52 \pm 11.78$ \\
$97.43 \pm 0.69$ \\
$93.22 \pm 7.22$ \\
$15.21 \pm 0.91$
\end{tabular}

$P$ value

value

Altitudes: PR, Pre expedition =550 m; BC1, Base Camp 1=4.844 m; C2, Camp2=6022 m; BC2, Base Camp 2 (after acclimatization) =4.844 m; C3, Camp3 $=7050 \mathrm{~m} ;$ PO, Post expedition $=550 \mathrm{~m}$.

$\mathrm{SaO}_{2}$, saturation arterial oxygen (\%); $\mathrm{paO}_{2}$, partial pressure of oxygen $(\mathrm{mmHg}) ; \mathrm{Hb}$, haemoglobin $(\mathrm{g} / \mathrm{L})$.

() 2018 The authors Published by Bioscientifica Ltd

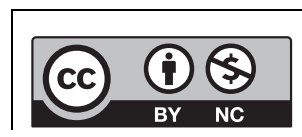

This work is licensed under a Creative Commons Attribution-NonCommercial 4.0 International License. 
Table 2 Cortisol concentrations ( $\mathrm{nmol} / \mathrm{L}$ ) at different altitude levels in the whole group of subjects and differences relative to PR.

\begin{tabular}{|c|c|c|c|c|c|}
\hline & \multirow[b]{2}{*}{ Mean \pm S.D. } & \multirow[b]{2}{*}{$n$} & \multicolumn{3}{|c|}{ Differences relative to $\mathrm{Pr}$} \\
\hline & & & Coefficient \pm S.E. & $P$ value & $95 \% \mathrm{Cl}$ \\
\hline PR & $474 \pm 163$ & 33 & & & \\
\hline $\mathrm{BC} 1$ & $369 \pm 106$ & 32 & $-110 \pm 28$ & 0.001 & $-165 ;-56$ \\
\hline $\mathrm{C} 2$ & $564 \pm 152$ & 13 & $48 \pm 38$ & 0.211 & $-27 ; 123$ \\
\hline $\mathrm{BC2}$ & $537 \pm 189$ & 24 & $51 \pm 30$ & 0.096 & $-9 ; 111$ \\
\hline $\mathrm{C} 3$ & $643 \pm 91$ & 7 & $161 \pm 49$ & 0.001 & $65 ; 257$ \\
\hline PO & $472 \pm 180$ & 34 & $-8 \pm 27$ & 0.757 & $-62 ; 45$ \\
\hline
\end{tabular}

Variation of cortisol concentrations were not sex, $\mathrm{SaO}_{2}$ or $\mathrm{paO}_{2}$ dependent.

Altitudes: PR, Pre expedition $=550 \mathrm{~m} ; \mathrm{BC}$, Base Camp $1=4.844 \mathrm{~m} ; \mathrm{C2}$ Camp2 $=6022 \mathrm{~m} ; \mathrm{BC2}$, Base Camp 2 (after acclimatization) $=4.844 \mathrm{~m} ; \mathrm{C3}$, Camp3 $=7050 \mathrm{~m} ;$ PO, Post expedition $=550 \mathrm{~m}$.

was not significantly different from baseline after the acclimatization period. Prolactin concentrations were sex but not $\mathrm{SaO}_{2}$ or $\mathrm{paO}_{2}$ dependent.

\section{Thyroid axis}

TSH (Table 4) concentrations were not different compared to baseline apart from $7050 \mathrm{~m}$ altitude at which TSH concentration were 1.7-fold higher. In contrast, fT4 (Fig. 2 and Table 5) increased constantly with each altitude from $12.7 \pm 1.5 \mathrm{pmol} / \mathrm{L}$ before the expedition to reach $16.1 \mathrm{pmol} / \mathrm{L}$ at $7050 \mathrm{~m}$. During acclimatization at $4844 \mathrm{~m}$, fT4 concentration decreased but stayed higher than baseline. These changes were less pronounced for fT3 (Table 6). At $4844 \mathrm{~m}$ fT3 concentrations also increased significantly, but at $6022 \mathrm{~m}$ and $7050 \mathrm{~m}$ altitude fT3 concentrations were similar to baseline. The thyroid axis was partly hypoxia dependent. TSH was $\mathrm{paO}_{2}$ and fT3 was $\mathrm{SaO}_{2}$ and $\mathrm{paO}_{2}$ dependent.

Table 3 Prolactin concentrations $(\mu \mathrm{g} / \mathrm{L})$ at different altitude levels in the whole group of subjects and differences relative to PR.

\begin{tabular}{|c|c|c|c|c|c|}
\hline & \multirow[b]{2}{*}{ Mean \pm s.D. } & \multirow[b]{2}{*}{$\boldsymbol{N}$} & \multicolumn{3}{|c|}{ Differences relative to $\operatorname{Pr}$ (adjusted for sex) } \\
\hline & & & Coefficient \pm S.E. & $P$ value & $95 \% \mathrm{Cl}$ \\
\hline PR & $9.1 \pm 4.1$ & 33 & & & \\
\hline $\mathrm{BC} 1$ & $6.9 \pm 4.9$ & 32 & $-2.2 \pm 0.6$ & $<0.001$ & $-3.4 ;-0.9$ \\
\hline $\mathrm{C} 2$ & $8.3 \pm 5.9$ & 13 & $-1.2 \pm 0.8$ & 0.143 & $-2.9 ; 0.4$ \\
\hline $\mathrm{BC} 2$ & $7.1 \pm 4.0$ & 23 & $-1.5 \pm 0.7$ & 0.031 & $-2.8 ;-0.1$ \\
\hline $\mathrm{C} 3$ & $5.4 \pm 1.1$ & 7 & $-2.4 \pm 1.1$ & 0.026 & $-4.5 ;-0.3$ \\
\hline PO & $8.5 \pm 3.6$ & 34 & $-0.5 \pm 0.6$ & 0.368 & $-1.7 ; 0.6$ \\
\hline
\end{tabular}

Variation of prolactin was sex $(P=0.015)$ but not $\mathrm{SaO}_{2}$ or $\mathrm{paO}_{2}$ dependent. Altitudes: PR, Pre expedition $=550 \mathrm{~m} ; \mathrm{BC} 1$, Base Camp $1=4.844 \mathrm{~m} ; \mathrm{C2}$, Camp2 $=6022 \mathrm{~m} ; \mathrm{BC2}$, Base Camp 2 (after acclimatization) $=4.844 \mathrm{~m} ; \mathrm{C3}$, Camp3 $=7050 \mathrm{~m} ;$ PO, Post expedition $=550 \mathrm{~m}$.
Table 4 TSH concentrations (mU/L) at different altitude levels in the whole group of subjects and differences relative to PR.

\begin{tabular}{|c|c|c|c|c|c|}
\hline & \multirow[b]{2}{*}{ Mean \pm S.D. } & \multirow[b]{2}{*}{$\boldsymbol{N}$} & \multicolumn{3}{|c|}{ Differences relative to $\mathrm{Pr}$ (adjusted for $\mathrm{paO}_{2}$} \\
\hline & & & Coefficient \pm S.E. & $P$ value & $95 \% \mathrm{Cl}$ \\
\hline PR & $2.15 \pm 1.33$ & 38 & & & \\
\hline BC1 & $2.27 \pm 1.22$ & 38 & $0.07 \pm 0.10$ & 0.471 & $-0.13 ; 0.28$ \\
\hline $\mathrm{C} 2$ & $2.20 \pm 1.26$ & 30 & $0.03 \pm 0.11$ & 0.770 & $-0.18 ; 0.25$ \\
\hline C3 & $3.46 \pm 2.88$ & 13 & $1.08 \pm 0.15$ & $<0.001$ & $0.79 ; 1.38$ \\
\hline $\mathrm{BC2}$ & $2.28 \pm 1.43$ & 27 & $0.08 \pm 0.11$ & 0.471 & $-0.14 ; 0.31$ \\
\hline $\mathrm{PO}$ & $1.93 \pm 1.09$ & 37 & $-0.20 \pm 0.10$ & 0.055 & $-0.40 ; 0.01$ \\
\hline
\end{tabular}

Variation of TSH was not sex and $\mathrm{SaO}_{2}$ but $\mathrm{PaO}_{2}(P=0.011)$ dependent Altitudes: PR, Pre expedition $=550 \mathrm{~m} ; \mathrm{BC1}$, Base Camp $1=4.844 \mathrm{~m} ; \mathrm{C2}$, Camp2 $=6022 \mathrm{~m}$; BC2, Base Camp 2 (after acclimatization) $=4.844 \mathrm{~m}$; C3, Camp3 $=7050 \mathrm{~m} ;$ PO, Post expedition $=550 \mathrm{~m}$.

\section{Gonadal axes}

FSH (Table 7) decreased from $5.8 \pm 5.9 \mathrm{mU} / \mathrm{L}$ before the expedition constantly with increasing altitude to reach $3.3 \pm 2.1 \mathrm{mU} / \mathrm{L}$ at $7050 \mathrm{~m}$, which corresponds with a $40 \%$ decrease. After acclimatization at $4844 \mathrm{~m}, \mathrm{FSH}$ concentrations normalized compared to baseline.

LH concentrations (Table 8) also decreased significantly by around 50\% between baseline and $7050 \mathrm{~m}$ altitude. However, the decrease could not be observed at all altitudes. At $6022 \mathrm{~m}$, there was an even smaller increase compared to $4844 \mathrm{~m}$. The normalizing effect of acclimatization could also be seen for LH. At $4844 \mathrm{~m}$, LH concentrations were significantly lower compared to baseline, an effect that was not recognizable anymore after acclimatization.

Testosterone concentrations were similar at all altitudes apart from $7050 \mathrm{~m}$ altitude. At this altitude, testosterone concentration was - in parallel to reduced concentrations of $\mathrm{LH}$ at that altitude - 50\% lower compared to baseline. A hypoxia dependency of the

Table 5 fT4 concentrations ( $\mathrm{pmol} / \mathrm{L}$ ) at different altitude levels in the whole group of subjects and differences relative to PR.

\begin{tabular}{|c|c|c|c|c|c|}
\hline & \multirow[b]{2}{*}{ Mean \pm S.D. } & \multirow[b]{2}{*}{$\mathbf{N}$} & \multicolumn{3}{|c|}{ Differences relative to $\mathrm{Pr}$} \\
\hline & & & Coefficient \pm S.E. & $P$ value & $95 \% \mathrm{Cl}$ \\
\hline PR & $12.7 \pm 1.5$ & 39 & & & \\
\hline $\mathrm{BC} 1$ & $15.2 \pm 2.2$ & 38 & $2.5 \pm 0.3$ & $<0.001$ & $2.0 ; 3.0$ \\
\hline $\mathrm{C} 2$ & $15.4 \pm 2.3$ & 31 & $2.7 \pm 0.3$ & $<0.001$ & $2.2 ; 3.3$ \\
\hline $\mathrm{C} 3$ & $16.1 \pm 2.1$ & 13 & $3.9 \pm 0.4$ & $<0.001$ & $3.1 ; 4.6$ \\
\hline $\mathrm{BC} 2$ & $14.1 \pm .2 .1$ & 28 & $1.5 \pm 0.3$ & $<0.001$ & $1.0 ; 2.1$ \\
\hline PO & $12.3 \pm 1.5$ & 38 & $-0.4 \pm 0.3$ & 0.1 .76 & $-0.9 ; 1.2$ \\
\hline
\end{tabular}

Variation of fT4 was not sex, $\mathrm{SaO}_{2}$ or $\mathrm{paO}_{2}$ dependent. Altitudes: PR, Pre expedition $=550 \mathrm{~m} ; \mathrm{BC} 1$, Base Camp $1=4.844 \mathrm{~m} ; \mathrm{C} 2$, Camp2 $=6022 \mathrm{~m} ; \mathrm{BC2}$, Base Camp 2 (after acclimatization) $=4.844 \mathrm{~m} ; \mathrm{C} 3$, Camp3 $=7050 \mathrm{~m}$; PO, Post expedition $=550 \mathrm{~m}$.

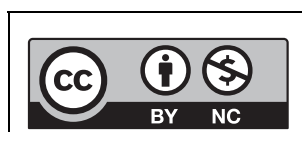

This work is licensed under a Creative Commons Attribution-NonCommercial 4.0 International License. 
Table 6 fT3 concentrations ( $p m o l / L$ ) at different altitude levels in the whole group of subjects and differences relative to PR.

\begin{tabular}{|c|c|c|c|c|c|}
\hline & \multirow[b]{2}{*}{ Mean \pm S.D. } & \multirow[b]{2}{*}{$n$} & \multicolumn{3}{|c|}{ Differences relative to $\mathrm{Pr}$ (adjusted for $\mathrm{SaO}_{2}$ ) } \\
\hline & & & Coefficient \pm S.E. & $P$ value & $95 \% \mathrm{Cl}$ \\
\hline PR & $4.57 \pm 0.60$ & 39 & & & \\
\hline BC1 & $5.26 \pm 0.73$ & 38 & $0.69 \pm 0.10$ & $<0.001$ & $0.50 ; 0.89$ \\
\hline $\mathrm{C} 2$ & $4.56 \pm 0.74$ & 31 & $0.02 \pm 0.10$ & 0.855 & $-0.19 ; 0.22$ \\
\hline $\mathrm{C} 3$ & $4.76 \pm 0.76$ & 13 & $0.21 \pm 0.14$ & 0.143 & $-0.07 ; 0.49$ \\
\hline BC2 & $4.74 \pm 0.56$ & 29 & $0.15 \pm 0.11$ & 0.169 & $-0.62 ; 0.36$ \\
\hline PO & $4.89 \pm 0.61$ & 38 & $0.28 \pm 0.10$ & 0.004 & $0.10 ; 0.47$ \\
\hline
\end{tabular}

Variation of fT3 was not sex but $\mathrm{SaO}_{2}(P=0.018)$ and $\mathrm{paO}_{2}(P=0.024)$ dependent.

Altitudes: PR, Pre expedition $=550 \mathrm{~m} ; \mathrm{BC} 1$, Base Camp $1=4.844 \mathrm{~m} ; \mathrm{C2}$, Camp2 $=6022 \mathrm{~m} ; \mathrm{BC2}$, Base Camp 2 (after acclimatization) $=4.844 \mathrm{~m} ; \mathrm{C} 3$, Camp3 $=7050 \mathrm{~m} ;$ PO, Post expedition $=550 \mathrm{~m}$

gonadal endocrine parameters could only be found for testosterone, which was $\mathrm{SaO}_{2}$ dependent.

In summary, adrenal, thyroid and gonadal axes as well as prolactin secretion were affected by increasing altitude. Adrenal axis and prolactin were first suppressed at $4844 \mathrm{~m}$ and then activated with increasing altitude; thyroid axis and gonadal axis were both directly activated respectively suppressed with increasing altitude. Acclimatisation at $4844 \mathrm{~m}$ led to normalization of adrenal and gonadal but not of thyroid axes.

\section{Discussion}

Our study revealed that adrenal and thyroid axes as well as prolactin secretion are activated, and male gonadal axis is suppressed at high altitude. The study also revealed that acclimatization at $4844 \mathrm{~m}$ led to normalization of adrenal and gonadal but not of thyroid axes.

Table 7 FSH concentrations (mU/L) at different altitude levels in the whole group of subjects and differences relative to PR.

\begin{tabular}{|c|c|c|c|c|c|}
\hline & \multirow[b]{2}{*}{ Mean \pm S.D. } & \multirow[b]{2}{*}{$n$} & \multicolumn{3}{|c|}{ Differences relative to $\mathrm{Pr}$} \\
\hline & & & Coefficient \pm S.E. & $P$ value & $95 \% \mathrm{Cl}$ \\
\hline PR & $5.81 \pm 5.95$ & 21 & & & \\
\hline $\mathrm{BC} 1$ & $4.77 \pm 5.64$ & 21 & $-1.10 \pm 0.44$ & 0.011 & $-1.96 ;-0.25$ \\
\hline $\mathrm{C} 2$ & $4.33 \pm 4.46$ & 17 & $-2.10 \pm 4.69$ & $<0.001$ & $-0.30 ;-1.19$ \\
\hline $\mathrm{C} 3$ & $3.38 \pm 2.10$ & 8 & $-1.66 \pm 0.62$ & 0.007 & $-2.87 ;-0.45$ \\
\hline $\mathrm{BC} 2$ & $6.00 \pm 6.58$ & 16 & $-0.66 \pm 0.48$ & 0.167 & $-1.59 ; 0.27$ \\
\hline PO & $6.03 \pm 5.89$ & 21 & $0.16 \pm 4.37$ & 0.712 & $-0.69 ; 1.01$ \\
\hline
\end{tabular}

Variation of FSH was not sex, $\mathrm{SaO}_{2}$ or $\mathrm{paO}_{2}$ dependent.

Altitudes: PR, Pre expedition $=550 \mathrm{~m} ; \mathrm{BC} 1$, Base Camp $1=4.844 \mathrm{~m} ; \mathrm{C2}$, Camp2 $=6022 \mathrm{~m} ; \mathrm{BC2}$, Base Camp 2 (after acclimatization) $=4.844 \mathrm{~m} ; \mathrm{C} 3$, Camp3 $=7050 \mathrm{~m} ; \mathrm{PO}$, Post expedition $=550 \mathrm{~m}$.
Table 8 LH concentrations ( $\mathrm{mU} / \mathrm{L}$ ) at different altitude levels in the whole group of subjects and differences relative to PR.

\begin{tabular}{|c|c|c|c|c|c|}
\hline & \multirow[b]{2}{*}{ Mean \pm S.D. } & \multirow[b]{2}{*}{$n$} & \multicolumn{3}{|c|}{ Differences relative to $\mathrm{Pr}$} \\
\hline & & & Coefficient \pm S.E. & $P$ value & $95 \% \mathrm{Cl}$ \\
\hline PR & $3.81 \pm 1.98$ & 18 & & & \\
\hline BC1 & $2.39 \pm 1.55$ & 19 & $-1.57 \pm 0.36$ & $<0.001$ & $-0.29 ;-0.85$ \\
\hline $\mathrm{C} 2$ & $3.00 \pm 1.52$ & 18 & $-1.45 \pm 0.49$ & 0.003 & $-2.41 ;-0.48$ \\
\hline C3 & $1.88 \pm 1.25$ & 6 & $-1.85 \pm 0.54$ & 0.001 & $-2.91 ;-0.79$ \\
\hline $\mathrm{BC} 2$ & $3.26 \pm 1.87$ & 14 & $0.79 \pm 0.39$ & 0.044 & $-1.57 ;-0.21$ \\
\hline PO & $3.60 \pm 1.59$ & 19 & $-0.34 \pm 0.37$ & 0.346 & $-1.04 ; 0.37$ \\
\hline
\end{tabular}

Variation of $\mathrm{LH}$ was not sex, $\mathrm{SaO}_{2}$ and $\mathrm{paO}_{2}$ dependent.

Altitudes: PR, Pre expedition $=550 \mathrm{~m} ; \mathrm{BC} 1$, Base Camp $1=4.844 \mathrm{~m} ; \mathrm{C2}_{\text {, }}$ Camp2 $=6022 \mathrm{~m} ; \mathrm{BC2}$, Base Camp 2 (after acclimatization) $=4.844 \mathrm{~m} ; \mathrm{C} 3$, Camp3 $=7050 \mathrm{~m} ; \mathrm{PO}$, Post expedition $=550 \mathrm{~m}$.

Previous studies have already analysed endocrine parameters at differentaltitudes but revealed heterogeneous or even controversial results, which might be due to the small study sizes or suboptimal study designs.

As we performed a study with a large study group with both male and female participants, with an analysis at various altitude levels up to $7050 \mathrm{~m}$ and under study conditions, which were standardized as much as possible, we assume that our study results are quite representative. However, our study design could not exclude all confounders, which a field study is confronted with. We therefore tried to include several hormone parameters for each analysed hormone axis to get a more representative and more comprehensive picture of the endocrine system.

The reason for our study was not only to better understand what kind of metabolic changes and adaptations mountaineers face at high altitude expeditions, we also hoped to understand if the endocrine systems contribute to the incapability of humans to live above around $5000 \mathrm{~m}$ altitude.

Table 9 Testosterone concentrations ( $\mathrm{nmol} / \mathrm{L}$ ) at different altitude levels in the whole group of subjects and differences relative to PR.

\begin{tabular}{|c|c|c|c|c|c|}
\hline & \multirow[b]{2}{*}{ Mean \pm S.D. } & \multirow[b]{2}{*}{$\boldsymbol{n}$} & \multicolumn{3}{|c|}{ Differences relative to $\operatorname{Pr}$ (adjusted for $\mathrm{SaO}_{2}$ ) } \\
\hline & & & Coefficient \pm S.E. & $P$ value & $95 \% \mathrm{Cl}$ \\
\hline PR & $21.5 \pm 10.1$ & 18 & & & \\
\hline BC1 & $21.1 \pm 13.6$ & 19 & $-0.5 \pm 2.5$ & 0.821 & $-5.6 ; 4.4$ \\
\hline $\mathrm{C} 2$ & $23.9 \pm 13.5$ & 8 & $1.8 \pm 3.4$ & 0.585 & $-4.8 ; 8.6$ \\
\hline $\mathrm{C} 3$ & $10.7 \pm 3.2$ & 6 & $-10.7 \pm 3.7$ & 0.007 & $-18.1 ;-3.3$ \\
\hline $\mathrm{BC} 2$ & $28.5 \pm 10.9$ & 14 & $7.5 \pm 2.7$ & 0.005 & $2.0 ; 12.9$ \\
\hline $\mathrm{PO}$ & $18.4 \pm 6.4$ & 19 & $-2.7 \pm 2.5$ & 0.285 & $-7.7 ; 2.2$ \\
\hline
\end{tabular}

Variation of testosterone was not sex or $\mathrm{paO}_{2}$ but $\mathrm{SaO}_{2}(P=0.28)$ dependent.

Altitudes: PR, Pre expedition $=550 \mathrm{~m}$; BC1, Base Camp $1=4.844 \mathrm{~m} ; \mathrm{C} 2$, Camp2 $=6022 \mathrm{~m} ; \mathrm{BC2}$, Base Camp 2 (after acclimatization) $=4.844 \mathrm{~m} ; \mathrm{C} 3$, Camp3 $=7050 \mathrm{~m} ; \mathrm{PO}$, Post expedition $=550 \mathrm{~m}$.

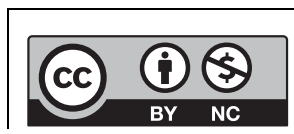

This work is licensed under a Creative Commons Attribution-NonCommercial 4.0 International License. 
Table 10 Pairwise comparisons of hormones shown in Tables $2,3,4,5,6,7,8$ and 9 ( $P$ values, bold $<0.05$ ).

\begin{tabular}{|c|c|c|c|c|c|c|c|c|}
\hline & $\begin{array}{c}\text { Cortisol } \\
\text { female and } \\
\text { male }\end{array}$ & $\begin{array}{c}\text { Prolactin } \\
\text { female and } \\
\text { male }\end{array}$ & $\begin{array}{l}\text { TSH female } \\
\text { and male }\end{array}$ & $\begin{array}{l}\text { fT4 female } \\
\text { and male }\end{array}$ & $\begin{array}{l}\text { fT3 female } \\
\text { and male }\end{array}$ & FSH male & LH male & Testosterone male \\
\hline Overall effect & $<0.001$ & 0.054 & $<0.001$ & $<0.001$ & $<0.001$ & $<0.001$ & $<0.001$ & $<0.001$ \\
\hline \multicolumn{9}{|l|}{ Ascent } \\
\hline BC1 vs PR & 0.001 & 0.016 & 0.240 & $<0.001$ & $<0.001$ & 0.159 & $<0.001$ & 0.751 \\
\hline C2 vs PR & 0.971 & 0.953 & 0.594 & $<0.001$ & 0.096 & $<0.001$ & 0.046 & 0.271 \\
\hline C3 vs PR & 0.015 & 0.464 & $<0.001$ & $<0.001$ & 0.009 & 0.100 & 0.009 & 1.000 \\
\hline$C 2$ vs $B C$ & 0.001 & 0.994 & 1.000 & 1.000 & 0.967 & 0.393 & 1.000 & 0.387 \\
\hline $\mathrm{C} 3$ vs $\mathrm{BC} 1$ & $<0.001$ & 1.000 & $<0.001$ & 0.009 & 1.000 & 0.999 & 1.000 & 0.997 \\
\hline $\mathrm{C} 3$ vs $\mathrm{C} 2$ & 0.484 & 0.999 & $<0.001$ & 0.076 & 0.864 & 1.000 & 1.000 & 0.018 \\
\hline \multicolumn{9}{|l|}{ Descent } \\
\hline $\mathrm{PO}$ vs $\mathrm{C} 3$ & 0.008 & 0.823 & $<0.001$ & $<0.001$ & 0.122 & 0.046 & 0.077 & 1.000 \\
\hline $\mathrm{PO}$ vs $\mathrm{C} 2$ & 0.887 & 1.000 & 0.008 & $<0.001$ & 0.652 & $<0.001$ & 0.275 & 0.104 \\
\hline PO vs BC2 & 0.536 & 0.940 & 0.980 & $<0.001$ & 1.000 & 0.746 & 0.985 & $<0.001$ \\
\hline \multicolumn{9}{|l|}{ Acclimatization } \\
\hline BC1 vs PR & 0.001 & 0.016 & 0.240 & $<0.001$ & $<0.001$ & 0.159 & $<0.001$ & 0.751 \\
\hline BC2 vs PR & 0.079 & 0.451 & 0.074 & $<0.001$ & 0.161 & 0.936 & 0.492 & 0.006 \\
\hline$B C 2$ vs $B C 1$ & $<0.001$ & 0.999 & 0.017 & 0.009 & $<0.001$ & 0.999 & 0.550 & 0.839 \\
\hline \multicolumn{9}{|c|}{ Long term effect } \\
\hline PO vs PR & 1.000 & 1.000 & 0.012 & 0.945 & 0.059 & 1.000 & 0.998 & 0.991 \\
\hline
\end{tabular}

Data are adjusted for sex (Prolactin), $\mathrm{SaO}_{2}$ (fT3, Testosterone) and $\mathrm{paO}_{2}$ (TSH). Sidak adjustment for multiple comparisons was considered.

It is well known that hypobaric hypoxia leads to several cardiorespiratory and metabolic changes (12), which preserve sufficient performance at higher altitude. This is impressively demonstrated by the ability of humans to ascend to over $7000 \mathrm{~m}$ above sea level without additional oxygen. However, staying at this altitude is only possible for a brief period of time. The critical altitude for living permanently is usually about $5000 \mathrm{~m}$, although this limit may vary depending on individual disposition and population group. The reasons for the limited ability of humans to live at a high altitude are not clear. It is known that a life-threatening form of altitude sickness can develop, which leads to physical degeneration and deterioration and is fatal. However, it is unclear what controls the metabolic changes at high altitude. This raises the question of whether these processes are also under endocrinological control.

Our study provides evidence for this hypothesis, as the adrenal axis is activated at high altitude, but can at least partially normalize at an altitude of about $5000 \mathrm{~m}$. The strong activation of the adrenal axis as well as the thyroid axis, and inhibition of the gonadal axes at very high altitude suggest that adaptation might not be longer possible at this altitude, and thus, might contribute to the physical degradation. However, it needs to be stressed that the endocrine changes might only reflect the adaptation of the body without significantly affecting the physical status at high altitude. The dysregulation of the endocrine system at high altitude is only mild to moderate and the

https://ec.bioscientifica.com
https://doi.org/10.1530/EC-18-0242

endocrine system might to some extent adapt to very high altitude.

Interestingly, the effects are not significantly different in men and women. We found similar effects in the adrenal and thyroid axes in both women and men. The gonadal axis could only be studied in men due to the

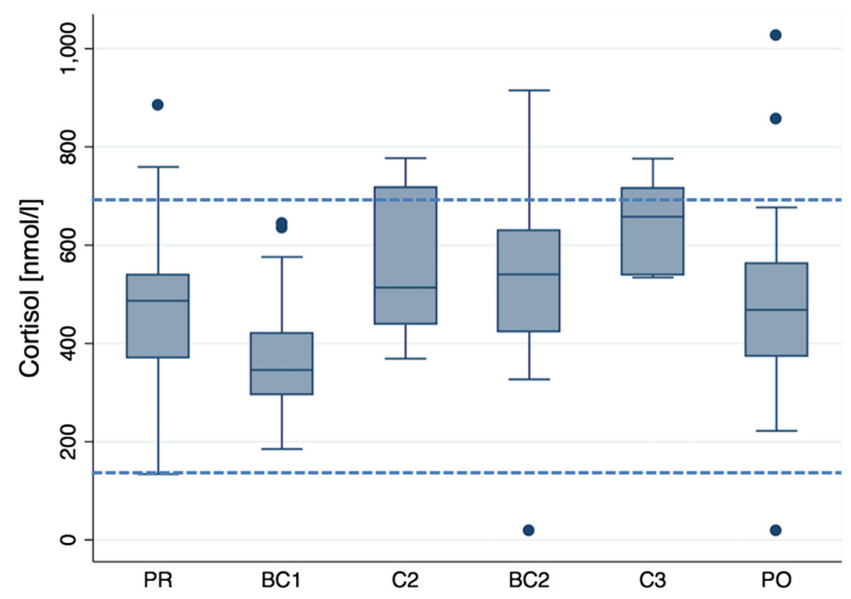

Figure 1

Cortisol concentration (nmol/L), representing a stress axis. Effect of altitude at $550 \mathrm{~m}$ before the expedition (PR), during ascent at $4.844 \mathrm{~m}$ (BC1), $6.022 \mathrm{~m} \mathrm{(C2)} \mathrm{and} 7.050 \mathrm{~m}$ altitude (C3), during descent at $4.822 \mathrm{~m}$ after acclimatization (BC2) and after the expedition at $550 \mathrm{~m}$ (P0). Boxes represent the 25th and 75th percentiles, and the bottom and top whiskers are defined by the 25th percentile minus 1.5 times the interquartile range (IQR) and the 75th percentile plus 1.5 times the IQR, respectively. Data points outside this range are plotted as individual points. Horizontal lines represent normal values. Statistical comparisons are shown in Tables 2 and 10.

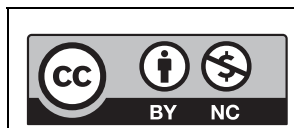
This work is licensed under a Creative Commons
Attribution-NonCommercial 4.0 International License. 


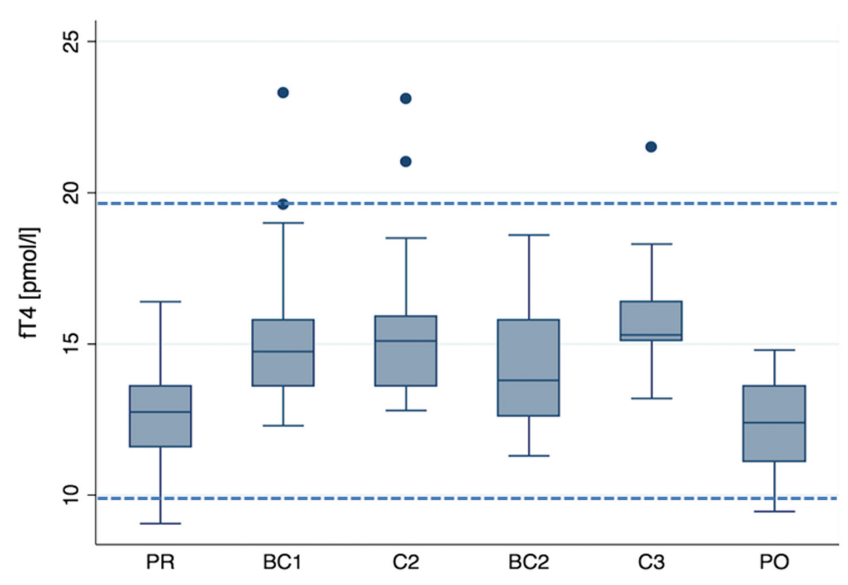

Figure 2

fT4 concentration (pmol/L), representing a thyroid axis. Legend: see Fig. 1. Statistical comparisons are shown in Tables 5 and 10.

cycle-related fluctuations in female gonadotrophins, which is a limitation of the study. It can be assumed, however, that suppression of the gonadal axis also occurs in women at high altitude, since the female gonadal axis is very sensitive to stressors and reacts with a dysregulated hypothalamic-pituitary pulsatility (13). We had also analysed the pulsatility of the hormone secretion to identify very subtle changes of the endocrine regulation, which will be published in a different paper.

Previous studies have already investigated the effect of hypobaric hypoxia on the endocrine system.

Cortisol concentrations appear to increase with increasing altitude, according to previous studies. Woods et al. showed a significant increase in resting cortisol concentrations at approximately $5000 \mathrm{~m}$ above sea level (8). Our study confirmed cortisol increase at high altitudes in both women and men. However, our study at $4844 \mathrm{~m}$ initially showed a cortisol decrease and a cortisol increase only at higher altitudes. Woods et al. also described lower cortisol levels below $5000 \mathrm{~m}$ (8). They found non-significantly lower concentrations at $4270 \mathrm{~m}$ compared to $5150 \mathrm{~m}$. Significantly lower concentrations were noted when exercise (6-h trek) took place before the measurement.

Prolactin concentrations were previously found to first decrease in men at $5380 \mathrm{~m}$, but then increase significantly if they stay at this altitude for several months (10). A significant increase in prolactin was also found in nine mountaineers in whom baseline prolactin levels were measured directly after climbing Mount Everest (5). Our study confirmed lower prolactin concentrations at $4844 \mathrm{~m}$ and elevated concentrations at $6022 \mathrm{~m}$ altitude. However, the increase was not significant.
Thyroid function dysregulations are not clear according to previous studies. Nepal et al. compared thyroid function in indigenous people at 2800 and $3750 \mathrm{~m}$ (9). fT4 was significantly elevated at $3750 \mathrm{~m}$, but not fT3 and TSH. Basu et al. found significantly higher fT3 and fT4 concentrations in soldiers after acclimatization at an altitude of about $6300 \mathrm{~m}$ (3). Hackney et al. found significantly lower TSH and fT3 concentrations in 15 mountaineers who had climbed Denali in Alaska (6194 m), whereas fT4 concentrations remained unchanged (2). In our study, the most striking differences were found for fT4, which increased with altitude. Results were not clear for fT3 and TSH, but overall, the thyroid axis was activated. As the thyroid gland releases mainly T4, which is metabolized to T3, and as T4 has a much longer half-life than T3, it can be speculated that the altitude adaptations of thyroid function are easier to be detected using T4 levels. TSH might also not be an ideal parameter for the detection of altitude-dependent functional changes as an increase in TSH may barely be detectable due to the negative feedback loop of T3 and T4 (14). Although a comparison of the studies is only possible to a limited extent and the data are somewhat heterogeneous, there appears to be an overall activation of the thyroid with increasing altitude, which is not detected at a pituitary level but in the thyroid gland.

Gonadal axes have previously poorly been studied. In the study by Benso et al., significantly reduced testosterone concentrations were found in eight mountaineers in the Base Camp at $5200 \mathrm{~m}$ directly after climbing Mount Everest (5); however, the interpretation of these results is limited due to the small number of test persons and excessive exertion. We found suppression of FSH and partly in LH with increasing altitude. Testosterone did not change very much but interestingly both LH and testosterone were reduced at $4800 \mathrm{~m}$. As LH stimulation regulates the production of testosterone (15), it can be assumed that low testosterone concentrations are a result of suppressed LH.

Acclimatization and its effects have not yet been investigated in other studies with a suitable study design. Only the effect of several months' stay at $5380 \mathrm{~m}$ (10) has been analysed so far. LH and total testosterone concentrations decreased significantly in 52 soldiers after 6 months at this altitude. The hormone levels increased again within the next 6 months. Sperm concentration, motility and vitality were also significantly reduced after 6 months at this altitude. Sperm concentration improved over the following 6 months, but not motility. After returning to lower altitudes, semen analysis normalized

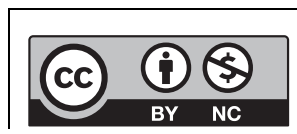

This work is licensed under a Creative Commons Attribution-NonCommercial 4.0 International License. 
again. The decrease of semen parameters at high altitude was potentially relevant for fertility. There was a moderate asthenozoospermia at altitude, corresponding with andrological subfertility. Six months after starting the altitude exposure, the overall motility was on average about $35 \%$ and after 12 months about $28 \%$. In our study, normalization of the stress axes in both sexes as well as the male gonadal axis after acclimatization was also demonstrated. We were able to show that normalization begins after just a few days.

All these study results raise the question of what causes these changes. Interestingly, the hormonal changes hardly correlated with parameters of oxygen transport such as $\mathrm{O}_{2}$ saturation and $\mathrm{pO}_{2}$ values, but only with altitude. Consequently, high-altitude hypoxia may not exclusively cause these changes, and other factors must also have an influence. The cold that occurs at high altitudes is probably not relevant or only of limited relevance because, due to good clothing, any relevant exposure to cold is mainly achieved via respiratory air. An effect of the increased physical burden at altitude due to hypobaric hypoxia is conceivable. 1, 8 demonstrated significantly higher cortisol levels or prolactin levels after physical exercise at an altitude of approximately $5000 \mathrm{~m}$. The effect of disturbed sleep due to periodic breathing at high altitudes would also be conceivable in terms of the change in stress axes (16). Insomnia and shortened sleep leads to significantly higher cortisol levels, even at low altitudes $(17,18)$. However, sleep does not improve with increasing acclimatization (16), and disturbed sleep at high altitude cannot fully explain the endocrine changes, as these, in contrast to sleep, partially normalize at $4800 \mathrm{~m}$. Because of this, it can be assumed that several height-dependent factors such as hypoxia, cold, physical stress, disturbed sleep, etc. have an influence and accumulate.

The question also arises if these endocrine changes might have a substantial effect on the health of humans living at high altitude for a long time. Insomnia leads to increased cortisol concentrations and to increased blood pressure and the risk for cardiovascular diseases (19). In line with this, patients with adverse cardiovascular events had higher median levels of cortisol (609.4 vs $594.4 \mathrm{pmol} /$ $\mathrm{mL}, \quad P<0.001)$ that those without (20). Clinical and subclinical hyperthyroidism is associated with different kinds of cardiovascular diseases (21). These effects might clinically be relevant as even a mild increase of blood pressure has an impact on mortality (22). The clinical consequences of high altitude on the gonadal axis has already been revealed by the above described studies on soldiers (10) which showed a clinically relevant reduction in sperm quality above about $5000 \mathrm{~m}$.

Therefore, in our view, the hypothesis is acceptable that the endocrine system might play a role in the fact that human beings cannot live long term at very high altitude.

In summary, the investigations show an activation of the adrenal and thyroid axes and inhibition of the male reproductive endocrine axis, which partially normalize at least at altitude of about $5000 \mathrm{~m}$. The endocrine changes found at higher altitude might contribute to the physical degradation and thereby to the incapability of humans to live permanently at very high altitude.

\section{Declaration of interest}

The authors declare that there is no conflict of interest that could be perceived as prejudicing the impartiality of the research reported.

\section{Funding}

The study was supported by the Swiss Mountain Medicine Society, Insel Foundation and Swisslos-Funds Canton Aargau.

\section{Author contribution statement}

$\mathrm{M} \vee \mathrm{W}$ and $\mathrm{J} \mathrm{P} \mathrm{H}$ designed the study, participated in the expedition, analysed the data and prepared the manuscript. M T prepared the samples and performed the analysis. C T N performed the statistical analysis. J D $\mathrm{V}$ provided support in the design of the study. T M M provided the vote of the cantonal ethics committee, organized and participated in the expedition. A R H performed the hormone analysis. All authors revised the final manuscript.

\section{Acknowledgements}

The authors would like to thank Dr Elizabeth Kraemer for the linguistic revision and correction of the manuscript. They are thankful to all the volunteers and greatly appreciate the support of the Swiss mountain guides, local guides and staff who made this expedition a successful one. Finally, the authors thank Nicole Bretschneider and Jana Bauer for their support in obtaining and preparing the blood samples during the expedition.

\section{References}

1 Utsunomiya T, Kadota T \& Yanaga T. Pituitary hormone responses to exercise at high altitudes. Nihon Naibunpi Gakkai Zasshi 198460 1214-1226.

2 Hackney AC, Feith S, Pozos R \& Seale J. Effects of high altitude and cold exposure on resting thyroid hormone concentrations. Aviation, Space, and Environmental Medicine 199566 325-329

3 Basu M, Pal K, Malhotra AS, Prasad R \& Sawhney RC. Free and total thyroid hormones in humans at extreme altitude. International Journal of Biometeorology 199539 17-21. (https://doi.org/10.1007/ BF01320888)

4 Basu M, Pal K, Prasad R, Malhotra AS, Rao KS \& Sawhney RC. Pituitary, gonadal and adrenal hormones after prolonged residence
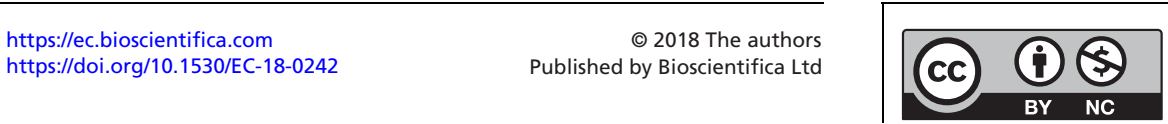
at extreme altitude in man. International Journal of Andrology 199720 153-158. (https://doi.org/10.1046/j.1365-2605.1997.00046.x)

5 Benso A, Broglio F, Aimaretti G, Lucatello B, Lanfranco F, Ghigo E \& Grottoli S. Endocrine and metabolic responses to extreme altitude and physical exercise in climbers. European Journal of Endocrinology 2007157 733-40.

6 Richalet JP, Letournel M \& Souberbielle JC. Effects of high-altitude hypoxia on the hormonal response to hypothalamic factors. American Journal of Physiology: Regulatory, Integrative and Comparative Physiology 2010299 R1685-R1692.

7 Riedl S, Kluge M, Schweitzer K, Waldhör T \& Frisch H. Adaptation of ghrelin and the GH/IGF axis to high altitude. European Journal of Endocrinology 2012166 969-976.

8 Woods DR, Davison A, Stacey M, Smith C, Hooper T, Neely D, Turner S, Peaston R \& Mellor A. The cortisol response to hypobaric hypoxia at rest and post-exercise. Hormone and Metabolic Research 201244 302-305. (https://doi.org/10.1055/s-0032-1304322)

9 Nepal O, Pokhrel BR, Khanal K, Gyawali P, Malik SL, Koju R \& Kapoor BK. Thyroid hormone levels in highlanders - a comparison between residents of two altitudes in Nepal. Kathmandu University Medical Journal 201311 18-21.

10 He J, Cui J, Wang R, Gao L, Gao X, Yang L, Zhang Q, Cao J \& Yu W. Exposure to hypoxia at high altitude $(5380 \mathrm{~m})$ for 1 year induces reversible effects on semen quality and serum reproductive hormone levels in young male adults. High Altitude Medicine and Biology 2015 16 216-222. (https://doi.org/10.1089/ham.2014.1046)

11 HiReach. (available at: http://www.pichler.ch/enc/). Last accessed June 2nd 2018.

12 Luks AM. Physiology in medicine: a physiologic approach to prevention and treatment of acute high-altitude illnesses. Journal of Applied Physiology 1985118 509-519. (https://doi.org/10.1152/ japplphysiol.00955.2014)

13 Warren MP \& Perlroth NE. The effects of intense exercise on the female reproductive system. Journal of Endocrinology. 2001170 3-11. (https://doi.org/10.1677/joe.0.1700003)

14 Roelfsema F, Boelen A, Kalsbeek A \& Fliers E. Regulatory aspects of the human hypothalamus-pituitary-thyroid axis. Best Practice and
Research: Clinical Endocrinology and Metabolism 201731 487-503. (https://doi.org/10.1016/j.beem.2017.09.004)

15 Sofikitis N, Giotitsas N, Tsounapi P, Baltogiannis D, Giannakis D $\&$ Pardalidis N. Hormonal regulation of spermatogenesis and spermiogenesis. Journal of Steroid Biochemistry and Molecular Biology 2008109 323-330. (https://doi.org/10.1016/j. jsbmb.2008.03.004)

16 Bloch KE, Latshang TD, Turk AJ, Hess T, Hefti U, Merz TM, Bosch MM, Barthelmes D \& Hefti JP, Maggiorini M, Schoch OD. Nocturnal periodic breathing during acclimatization at very high altitude at Mount Muztagh Ata (7,546 m). American Journal of Respiratory and Critical Care Medicine 2010182 562-568. (https://doi. org/10.1164/rccm.200911-1694OC)

17 Vgontzas AN, Bixler EO, Lin HM, Prolo P, Mastorakos G, VelaBueno A, Kales A \& Chrousos GP. Chronic insomnia is associated with nyctohemeral activation of the hypothalamic-pituitaryadrenal axis: clinical implications. Journal of Clinical Endocrinology and Metabolism. 200186 3787-3794. (https://doi.org/10.1210/ jcem.86.8.7778)

18 D'Aurea C, Poyares D, Piovezan RD, Passos G, Tufik S \& Mello MT Objective short sleep duration is associated with the activity of the hypothalamic-pituitary-adrenal axis in insomnia. Arquivos de NeuroPsiquiatria 201573 516-519.

19 Khan MS \& Aouad R. The effects of insomnia and sleep loss on cardiovascular disease. Sleep Medicine Clinics 201712 167-177. (https://doi.org/10.1016/j.jsmc.2017.01.005)

20 Jutla SK, Yuyun MF, Quinn PA \& Ng LL. Plasma cortisol and prognosis of patients with acute myocardial infarction. Journal of Cardiovascular Medicine 201415 33-41. (https://doi.org/10.2459/ JCM.0b013e328364100b)

21 Faber J \& Selmer C. Khan cardiovascular disease and thyroid function. Frontiers of Hormone Research 201443 45-56.

22 Vaduganathan M, Pareek M, Quamar A, Pandey A, Olsen MH \& Bhatt DL. Baseline blood pressure, the 2017 ACC/AHA high blood pressure guidelines, and long-term cardiovascular risk in SPRINT. American Journal of Medicine 2018131 956-960. (https://doi. org/10.1016/j.amjmed.2017.12.049)

\section{Received in final form 22 August 2018}

Accepted 29 August 2018

Accepted Preprint published online 31 August 2018 https://ec.bioscientifica.com https://doi.org/10.1530/EC-18-0242 (c) 2018 The authors Published by Bioscientifica Ltd

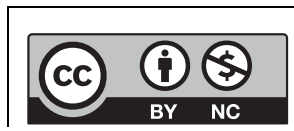

This work is licensed under a Creative Commons Attribution-NonCommercial 4.0 International License. 